\title{
Cross-Correlated Responses of Topological Superconductors and Superfluids
}

\author{
Kentaro Nomura, ${ }^{1}$ Shinsei Ryu, ${ }^{2,3}$ Akira Furusaki, ${ }^{3}$ and Naoto Nagaosa ${ }^{1,4,5}$ \\ ${ }^{1}$ Correlated Electron Research Group (CERG), RIKEN-ASI, Wako 351-0198, Japan \\ ${ }^{2}$ Department of Physics, University of Illinois, 1110 West Green St, Urbana IL 61801, USA \\ ${ }^{3}$ Condensed Matter Theory Laboratory, RIKEN, Wako, Saitama 351-0198, Japan \\ ${ }^{4}$ Cross-Correlated Material Research Group (CMRG), RIKEN-ASI, Wako 351-0198, Japan \\ ${ }^{5}$ Department of Applied Physics, The University of Tokyo, Hongo, Bunkyo-ku, Tokyo 113-8656, Japan
}

(Dated: August 29, 2018)

\begin{abstract}
We study nontrivial responses of topological superconductors and superfluids to the temperature gradient and rotation of the system. In two-dimensional gapped systems, the Strěda formula for the electric Hall conductivity is generalized to the thermal Hall conductivity. Applying this formula to the Majorana surface states of three-dimensional topological superconductors predicts cross-correlated responses between the orbital angular momentum and thermal polarization (entropy polarization). These results can be naturally related to the gravitoelectromagnetism description of three-dimensional topological superconductors and superfluids, analogous to the topological magnetoelectric effect in $\mathbb{Z}_{2}$ topological insulators.
\end{abstract}

PACS numbers: 73.43.-f, 74.25.fc, 74.90.+n, 74.25.F-

Introduction The quantum Hall effect (QHE) [1] is a prominent example of quantum phenomena characteristic of insulators with topologically nontrivial electronic wave functions, a class of materials called topological insulators (TIs) [2]. In the QHE the Hall conductivity is quantized in units of $e^{2} / h$ at integer values equal to the topological number of bulk wave functions [3]. The two-dimensional (2d) topological superconductors (TSCs) and superfluids (TSFs) with chiral ( $p$-wave) Cooper pairing are superconductor analogues of the QHE and considered to be realized, e.g., in a thin film of ${ }^{3} \mathrm{He}$ A phase [4, 5], $\mathrm{Sr}_{2} \mathrm{RuO}_{4}$ [], and the $\nu=5 / 2$ fractional QHE [7. The topological nature of such TSCs and TSFs will manifest itself in thermal transport properties, such as quantization of the thermal Hall conductivity [8].

Recent studies have shown that topological states exist in time-reversal invariant and three-dimensional (3d) cases as well [2], and the systematic classification of them is established in terms of symmetries and dimensionality [9, 10]. A key experimental signature of 3d-TIs is the topological magnetoelectric (ME) effect. Namely, the electromagnetic response of 3d-TIs is described by the axion electrodynamics [2, 11, 12],

$$
S_{\theta}^{\mathrm{EM}}=\int d t d^{3} \boldsymbol{x} \frac{e^{2}}{4 \pi^{2} \hbar c} \theta \boldsymbol{E} \cdot \boldsymbol{B}
$$

with $\theta=\pi$, the possible nonzero value in time-reversal invariant systems $(\bmod 2 \pi)$. The effective action (11) leads to the surface QHE that induces the topological ME effect as $\boldsymbol{M}=\left(e^{2} / 2 h c\right) \boldsymbol{E}$ and $\boldsymbol{P}=\left(e^{2} / 2 h c\right) \boldsymbol{B}$ where $\boldsymbol{M}$ and $\boldsymbol{P}$ are the magnetization and electric polarization, respectively.

An example of 3d-TSFs 13] is the B phase of superfluid ${ }^{3} \mathrm{He}$ [9]. In addition, the newly found superconducting phase in $\mathrm{Cu}$-doped $\mathrm{Bi}_{2} \mathrm{Se}_{3} 14$ has been proposed to be a 3d-TSC 15]. The topological nature of 3d-TSCs manifests itself in their coupling with the gravitational field, which is described by the term similar to Eq. (1), the gravitational instanton term [16, 17].

In this work we shall develop the response theory of TSCs, which reveals the cross-correlation between thermal and mechanical (rotational) responses, and is the direct equivalent of the topological ME effect in TIs. We first generalize the Strěda formula 18 to the thermal Hall conductivity in $2 \mathrm{~d}$ systems. By applying it to the surface states of a 3d-TSC, the cross-correlated responses
$2 d$
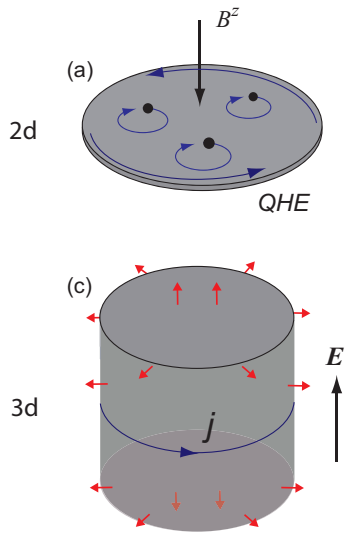

$3 d-T I$
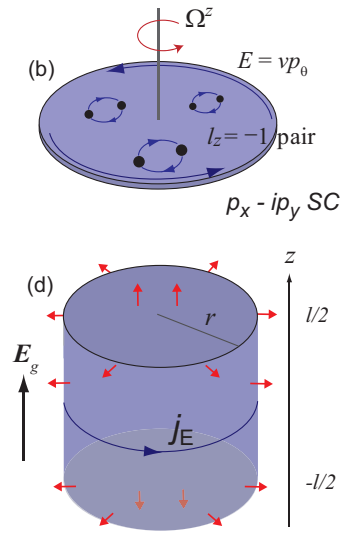

$3 d-T S C$
FIG. 1. (color online) Electronic responses in (a) twodimensional (2d) and (c) three-dimensional (3d) topological insulators (TIs) (left) and thermal and mechanical (rotating) responses in (b) 2d and (d) 3d topological superconductors (TSCs) (right). In (b), the arrow along the boundary indicates the chiral Majorana edge channel with dispersion $E=v p_{\theta}$. In the $3 \mathrm{~d}-\mathrm{TSC}(\mathrm{d})$, temperature gradient induces surface thermal Hall current $\boldsymbol{j}_{E}$. A uniform mass gap is induced in the surface fermion spectrum by doping magnetic impurities near the surface of the 3d-TI and TSC such that spins are all pointing out or in (red arrows). 


\begin{tabular}{|c|c|c|}
\hline & $\mathrm{TI}$ & $\mathrm{TSC}$ \\
\hline $2 \mathrm{~d}$ & $\sigma_{H}=e c \frac{\partial M^{z}}{\partial \mu}=e c \frac{\partial N}{\partial B^{z}}$ & $\kappa_{H}=\frac{v^{2}}{2} \frac{\partial L^{z}}{\partial T}=\frac{v^{2}}{2} \frac{\partial S}{\partial \Omega^{z}}$ \\
\hline $3 \mathrm{~d}$ & $\chi_{\theta}^{a b}=\frac{\partial M^{a}}{\partial E^{b}}=\frac{\partial P^{a}}{\partial B^{b}}$ & $\chi_{\theta, g}^{a b}=\frac{\partial L^{a}}{\partial E_{g}^{b}}=\frac{\partial P_{E}^{a}}{\partial \Omega^{b}}$ \\
\hline
\end{tabular}

TABLE I. Comparison between cross-correlation in topological insulators (TIs) and topological superconductors (TSCs) in two $(2 \mathrm{~d})$ and three spatial dimensions (3d). In TSCs the orbital angular momentum $\boldsymbol{L}$ and entropy $S$ (thermal polarization $\boldsymbol{P}_{E}$ in $3 \mathrm{~d}$ ) are generated by temperature gradient $\left(\boldsymbol{E}_{g}=-T^{-1} \nabla T\right)$ and by rotating the system with angular velocity $\Omega^{a}$. In analogy with the magnetoelectric polarizability $\chi_{\theta}^{a b}$ in $3 \mathrm{~d}$ TI gravitomagnetoelectric polarizability $\chi_{\theta, g}^{a b}$ can be introduced in the $3 \mathrm{~d}$ TSC (Right-bottom). Note that the relations for TSCs hold also for the thermal response of TIs. These responses are characterized by the topological integers and quantized. See Eq. (6) for 2d-TSCs and Eq. (10) for the surface of 3d-TSCs

of 3d-TSCs are identified with those between the orbital angular momentum and the thermal (entropy) polarization, generated by temperature gradient $\nabla T$ and by rotating the system with angular velocity $\boldsymbol{\Omega}$, respectively, as shown in Fig. 11. Our main findings are summarized in Table 1 Their derivations will be given below.

Thermal Hall conductivity Relation between the Hall conductivity $\sigma_{H}$ and the magnetization $\boldsymbol{M}$ in the quantum Hall regime is known as the Strěda formula [18]:

$$
\sigma_{H}=e c \frac{\partial M^{z}}{\partial \mu},
$$

where $\mu$ is the chemical potential, $e(<0)$ is the electric charge, and $c$ is the speed of light. The magnetization is evaluated by $\boldsymbol{M}=(e / 2 c) \operatorname{Tr}[\theta(\mu-\mathcal{H}) \boldsymbol{x} \times \boldsymbol{v}]$, where $\boldsymbol{v}=(i / \hbar)[\mathcal{H}, \boldsymbol{x}], \mathcal{H}$ is the single-particle Hamiltonian, and $\theta(x)$ is the step function. The relation (2) can be understood by identifying the Hall current $\boldsymbol{j}=\sigma_{H} \boldsymbol{E} \times \hat{\mathbf{z}}$ with $\boldsymbol{j}=c \nabla \times \boldsymbol{M}=-c \partial \boldsymbol{M} / \partial \mu \times \nabla \mu$.

For the thermal conductivity $\kappa_{H}=j_{T}^{x} / \partial_{y} T$, we will show below that a similar relation holds:

$$
\kappa_{H}=c \frac{\partial M_{T}^{z}}{\partial T} .
$$

The thermal current is $\boldsymbol{j}_{T}=\boldsymbol{j}_{E}-(\mu / e) \boldsymbol{j}$, where $\boldsymbol{j}$ is the electric charge current. The energy-current $\boldsymbol{j}_{E}$ is defined as $j_{E}^{a}=c T^{a 0}$, where $T^{a 0}$ is a spatio-temporal component of the energy-momentum tensor, $T^{\mu \nu}=$ $\left(\delta \mathcal{L} / \delta \partial_{\mu} \Psi\right) \partial^{\nu} \Psi-g^{\mu \nu} \mathcal{L}$, satisfying the continuity equation, $\partial_{\mu} T^{\mu \nu}=0 ; \mathcal{L}\left[\Psi, \partial_{\mu} \Psi\right]$ is the Lagrangian density of the system. The moment of the thermal current in Eq. (3) is defined as $\boldsymbol{M}_{T}=\boldsymbol{M}_{E}-(\mu / e) \boldsymbol{M}$, where $M_{E}^{\mu \nu}=\frac{1}{2}\left\langle x^{\mu} T^{\nu 0}-x^{\nu} T^{\mu 0}\right\rangle$, and $M_{E}^{z}=M_{E}^{12}$. In contrast to the charge Hall conductivity (2), the average has to be taken at finite temperature: $\langle\cdots\rangle \equiv \sum_{n} f\left(\varepsilon_{n}\right)\langle n|\cdots| n\rangle$ where $\varepsilon_{n}$ and $|n\rangle$ are eigenvalue and eigenstate of the Hamiltonian $\mathcal{H}$, and $f\left(\varepsilon_{n}\right)$ is the Fermi distribution function. Below, we will consider the part of the thermal current carried by $\boldsymbol{j}_{E}$, specializing to the case of $\mu=0$. For insulators, this means we count the total number $N$ of particles such that when the chemical potential is within a gap, $N=0$. In the Bogoliubov-de Genne theory of superconductors, due to particle-hole symmetry, this is always true, and hence $\boldsymbol{j}_{T}=\boldsymbol{j}_{E}$ and $\boldsymbol{M}_{T}=\boldsymbol{M}_{E}$.

Let us now introduce a gravitomagnetic field $\boldsymbol{B}_{g}$ 19] which is conjugate to $\boldsymbol{M}_{E}$ so that the variation of free energy is $d F=-S d T-\boldsymbol{M}_{E} \cdot d \boldsymbol{B}_{g}$. Equation (3) is written as $\kappa_{H}=c\left(\partial M_{E}^{z} / \partial T\right)_{B_{g}^{z}}=c\left(\partial S / \partial B_{g}^{z}\right)_{T}$, or

$$
\kappa_{H}=\frac{c}{T}\left(\frac{\partial M_{E}^{z}}{\partial \phi}\right)_{B_{g}^{z}}=\frac{c}{T}\left(\frac{\partial Q}{\partial B_{g}^{z}}\right)_{\phi}
$$

by introducing $d Q=T d S$ and $d \phi=d T / T$, where $\phi$ is a fictitious gravitational potential that couples to thermal energy density $Q$ [20]. Equation (4) is the thermal analogue of the Strěda formula for the charge Hall conductivity, $\sigma_{H}=e c \partial M^{z} / \partial \mu=e c \partial N / \partial B^{z}$, in that $Q$ is the zeroth component of the energy current as $e N$ is in the charge current.

To see the physical meaning of $\boldsymbol{B}_{g}$ and $\boldsymbol{M}_{E}$, note that the definition of $M_{E}^{\mu \nu}$ is similar to the orbital angular momentum: $L^{\mu \nu}=(1 / c)\left\langle x^{\mu} T^{0 \nu}-x^{\nu} T^{0 \mu}\right\rangle$. Indeed, when there is a relativistic invariance, the energy-momentum tensor can be symmetrized so that $T^{\mu \nu}=T^{\nu \mu}$, and thus $M_{E}^{\mu \nu}=(c / 2) L^{\mu \nu}$. While this is not the case in condensed-matter systems in general, in so-called pseudorelativistic systems where the Lorentz invariance is realized at low energies, electrons or quasiparticles obey the Dirac or Majorana equation. In these systems the Fermi velocity $v$ plays a role of the speed of light $c$, and the $M_{E}^{\mu \nu}$ tensor is related to the orbital angular momentum as $\boldsymbol{M}_{E}=(v / 2) \boldsymbol{L}$, and $\boldsymbol{B}_{g}$ can be understood as the angular velocity vector of rotating systems, $\boldsymbol{B}_{g}=(2 / v) \boldsymbol{\Omega}$. For a system rotating with the frequency $\boldsymbol{\Omega}=\Omega^{z} \hat{\mathbf{z}}$, this can also be understood by making a coordinate transformation from the rest frame to the rotating frame in which the metric in the polar coordinates $(t, r, \varphi)$ takes the form $d s^{2} \simeq v^{2} d t^{2}-2 \Omega^{z} r^{2} d \varphi d t-r^{2} d \varphi^{2}-d r^{2}$. One can then read off, from the definition of the gravitoelectromagnetic field, the non-zero gravito gauge potential $A_{g}^{\varphi}=\Omega^{z} r / v$ 19. In Cartesian coordinates, $\boldsymbol{A}_{g}=(1 / v) \Omega^{z} \hat{\mathbf{z}} \times \boldsymbol{x}$, and $\boldsymbol{B}_{g}=\nabla \times \boldsymbol{A}_{g}=(2 / v) \Omega^{z} \hat{\mathbf{z}}$. Consequently, $\kappa_{H}$ can be written as

$$
\kappa_{H}=\frac{v^{2}}{2}\left(\frac{\partial L^{z}}{\partial T}\right)_{\Omega^{z}}=\frac{v^{2}}{2}\left(\frac{\partial S}{\partial \Omega^{z}}\right)_{T} .
$$

This is one of the main results of this work.

Although it is necessary to have (pseudo) Lorentz invariance to identify, at the operator level, $\boldsymbol{M}_{E}$ with the 
angular momentum $\boldsymbol{L}$, the relation (5) can in fact be derived for a wider range of systems, e.g., by assuming that the edge state in the disk geometry is described by chiral conformal field theory [21]. This indicates the validity of Eq. (15) in many $2 \mathrm{~d}$ topological phases, a representative example of which is the $2 \mathrm{~d}$ chiral $p$-wave $\mathrm{SC}$ with $l_{z}=-1$ pairing as shown in Fig. 1(b). Near the edge, there exist chiral Majorana modes $\|$ described by $H_{\text {edge }}=(1 / 2) \int_{0}^{L} d x \psi\left(-i \hbar v \partial_{x}\right) \psi$, where $\psi$ is a singlecomponent real fermionic field and $L$ is the circumference of the edge. Since $T^{00}=T^{10}=T^{01}=(-i \hbar v / 2) \psi \partial_{x} \psi$, $\left\langle j_{E}\right\rangle=(v / L)\left\langle H_{\text {edge }}\right\rangle=\left(v^{2} / 2\right)\left\langle L_{\text {edge }}^{z}\right\rangle=\pi^{2} k_{B}^{2} T^{2} / 12 h$, leading to

$$
\kappa_{H}=\frac{\partial\left\langle j_{E}\right\rangle}{\partial T}=\frac{\pi^{2} k_{B}^{2} T}{6 h},
$$

which is a half of the quantized value for conventional chiral fermions. Here, $L_{\text {edge }}^{z}$ is the edge modes contribution to the total orbital angular momentum per unit area.

Majorana fermion on the surface of 3d-TSC/TSF Let us now derive Eqs. (45) from a microscopic theory via Kubo formula. We work with an example, the $2 \mathrm{~d}$ massive Majorana fermion system realized on the surface of 3d-TSCs [2, 9, 22], anticipating to apply (4.5) to derive the cross-correlated responses of the 3d-TSCs. It is described by $H=(1 / 2) \int d^{2} \boldsymbol{x} \psi^{T} \mathcal{H} \psi$, where $\psi^{T}=\left(\psi_{\uparrow}, \psi_{\downarrow}\right)$ is the real spinor field satisfying $\left\{\psi_{\alpha}(\boldsymbol{x}), \psi_{\beta}\left(\boldsymbol{x}^{\prime}\right)\right\}=\delta_{\alpha \beta} \delta\left(\boldsymbol{x}-\boldsymbol{x}^{\prime}\right)$, and

$$
\mathcal{H}=-i \hbar v\left(\sigma_{z} \partial_{x}+\sigma_{x} \partial_{y}\right)+m \sigma_{y} .
$$

The mass $m$ is due to the interaction with magnetic fields or magnetic moments perpendicular to the surface [Fig. [1(d)] and thus breaks time-reversal symmetry. To study thermal transport, we introduce coupling with the fictitious gravitational potential $\phi$ in the Lagrangian 20, 23: $\mathcal{L}=(1 / 2) \psi^{T}\left[i \hbar \partial_{t}-\mathcal{H}-(1 / 2)\{\phi, \mathcal{H}\}\right] \psi$. The energycurrent operator is given by $(a, b=x, y)$

$$
\begin{aligned}
j_{E}^{a}= & \psi^{T} \frac{1}{4}\left\{v^{a}, \mathcal{H}\right\} \psi-\psi^{T} \frac{i \hbar}{8}\left[v^{a}, v^{b}\right] \psi \partial_{b} \phi \\
& +\psi^{T} \frac{1}{8}\left[\mathcal{H}\left(v^{a} x^{b}+3 x^{b} v^{a}\right)+\text { H.c. }\right] \psi \partial_{b} \phi .
\end{aligned}
$$

The first term is non-vanishing even in the absence of gravitational potential; the second and the third terms are proportional to $\nabla \phi$, which are analogous to the diamagnetic charge current in the presence of a magnetic field. To evaluate $\kappa_{H}=-\left\langle j_{E}^{x}\right\rangle /\left(T \partial_{y} \phi\right)$, we apply the Kubo linear response formula to the first term, while the second and third terms need to be averaged in thermal equilibrium [21]. When the mass gap is large enough, the density of states of Majorana fermions vanishes at $\varepsilon=0$, and the thermal Hall conductivity is given by $\kappa_{H}=$ $v \partial M_{E}^{z} / \partial T$, where $M_{E}^{z}=(1 / 4 v) \sum_{n} f\left(\varepsilon_{n}\right) \varepsilon_{n}\langle n|\left(x v^{y}-\right.$ $\left.y v^{x}\right)|n\rangle$, and $\langle\boldsymbol{x} \mid n\rangle=u_{n}(\boldsymbol{x})$ is the exact eigenstate of the Majorana Hamiltonian (7), $\mathcal{H} u_{n}(\boldsymbol{x})=\varepsilon_{n} u_{n}(\boldsymbol{x})$.
Quite generally, one can derive, in the limit $T \rightarrow 0$, the relation

$$
\kappa_{H}=\frac{\hbar \pi^{2} k_{B}^{2} T}{6 L^{2}} \sum_{n, m} \theta\left(-\epsilon_{n}\right) \frac{2 \operatorname{Im}\left[\left\langle n\left|v^{x}\right| m\right\rangle\left\langle m\left|v^{y}\right| n\right\rangle\right]}{\left(\epsilon_{n}-\epsilon_{m}\right)^{2}},
$$

where $L^{2}$ is the area of the surface. Apart from the factor $\pi^{2} k_{B}^{2} T / 6$, the right hand side resembles the Kubo formula for the electrical Hall conductivity, which, however, is not a well-defined quantity for Majorana fermions; nevertheless Eq. (9) can be regarded as the generalized Wiedemann-Franz law to Majorana fermions [4, 5, 20, 23]. Compared to the electron systems, there is an extra factor of $1 / 2$ due to Majorana nature. Since $\sigma_{H}$ of the massive Dirac fermion is $\sigma_{H}=\operatorname{sgn}(m) e^{2} /(2 h)$, Eq. (9) immediately gives

$$
\kappa_{H}=\operatorname{sgn}(m) \frac{\pi^{2}}{6} \frac{k_{B}^{2}}{2 h} T
$$

for the massive Majorana fermion. There is a factor $1 / 2$ compared to the $2 \mathrm{~d}$ result Eq. (6).

Cross-correlated response of 3d-TSC/TSF Let us illustrate physical implications of Eq. (5) by studying the responses of 3d-TSCs to the temperature gradient and the rotation. For simplicity, we consider a sample with cylindrical geometry with height $\ell$ and radius $r$ as depicted in Fig. 1(d). We assume that magnetic impurities are doped near the surface and their spins are all pointing out or in so that uniform mass gap is formed on the surface. Let us first introduce the temperature gradient in the $z$-direction, which generates the energy-current $j_{E}=\kappa_{H} \partial_{z} T$ on the surface. Since $j_{E} / v^{2}$ corresponds to the momentum per unit area, total momentum due to the surface energy-current is $P_{\varphi}=(2 \pi r \ell) j_{E} / v^{2}$ and thus the induced orbital angular momentum per volume is given by

$$
\left.L^{z}\right|_{\Omega^{z}}=\frac{r P_{\varphi}}{\pi r^{2} \ell}=\frac{2}{v^{2}} \kappa_{H} \partial_{z} T .
$$

Similarly, upon rotating the cylinder with $\Omega=\Omega^{z} \hat{\mathbf{z}}$ (without temperature gradient), applying Eqs. (田) and (5) to the top and bottom surfaces, we obtain the induced thermal energy density (the induced entropy change) localized on the top and bottom surfaces,

$$
\left.\Delta Q(z)\right|_{T}=\frac{2 T \Omega^{z}}{v^{2}}\left[\kappa_{H}^{\mathrm{t}} \delta(z-\ell / 2)+\kappa_{H}^{\mathrm{b}} \delta(z+\ell / 2)\right],
$$

where $\kappa_{H}^{\mathrm{t}}=-\kappa_{H}^{\mathrm{b}}$ as the spins on the top and bottom surfaces are pointing to the opposite directions (different signs of $m$ ); see Fig. 11(d) 24].

In terms of the gravitoelectric field $\boldsymbol{E}_{g}=-T^{-1} \nabla T$ and the momentum of the energy current $\boldsymbol{M}_{E}$, Eq. (11) can be written as $\boldsymbol{M}_{E}=\left(T \kappa_{H} / v\right) \boldsymbol{E}_{g}$. Further introducing the thermal polarization $\boldsymbol{P}_{E}$ by $\Delta Q=-\nabla \cdot \boldsymbol{P}_{E}$, Eq. (12) can be written similarly as $\boldsymbol{P}_{E}=\left(T \kappa_{H} / v\right) \boldsymbol{B}_{g}$. These 
highlight the correspondence between TIs and TSCs,

$$
\text { TI: } \frac{\partial M^{a}}{\partial E^{b}}=\frac{\partial P^{a}}{\partial B^{b}} \Leftrightarrow \text { TSC: } \frac{\partial M_{E}^{a}}{\partial E_{g}^{b}}=\frac{\partial P_{E}^{a}}{\partial B_{g}^{b}} .
$$

Since the orbital angular momentum is given from the internal energy functional by $L^{a}=-\delta U_{\theta} / \delta \Omega^{a}$, the coupling energy of the temperature gradient and rotation velocity is written as

$$
U_{\theta}=-\int d^{3} \boldsymbol{x} \frac{2}{v^{2}} \kappa_{H} \nabla T \cdot \boldsymbol{\Omega}=\int d^{3} \boldsymbol{x} \frac{k_{B}^{2} T^{2}}{24 \hbar v} \theta \boldsymbol{E}_{g} \cdot \boldsymbol{B}_{g} .
$$

This is analogous to Eq. (11) with $e^{2} / \hbar c \leftrightarrow\left(\pi k_{B} T\right)^{2} / 6 \hbar v$ and $\theta= \pm \pi$ playing the same role as in Eq. (1). In $2 \mathrm{~d}-$ TSC cases, the corresponding term is written as $U_{\text {TSC }}^{2 d}=$ $\int d^{2} x\left(2 / v^{2}\right) T \kappa_{H} \phi \Omega^{z}$. This is the thermodynamical analogue of the Chern-Simons term 2, 5, 25.

Angular momentum paradox in 2d-TSC/TSF These terms can be related to the problem of total angular momentum of the ground state of chiral superfluids. For simplicity we consider the $2 \mathrm{~d}$ case. The angular momentum per unit area of the ground state of the chiral $p$-wave state has been proposed to behave as $L^{z}(T=0)=-(\hbar n / 2)\left(k_{B} T_{c} / E_{F}\right)^{\gamma}$ with different exponents $\gamma=2,1$ and 0 细, where $n$ is the number of particles. The controversy of $\gamma$ has not yet resolved. By integrating Eq. (5) from 0 to $T$, and using $\Delta=\hbar v k_{F}$, one obtains $L^{z}(T)-L^{z}(0)=\left(\pi \hbar k_{F}^{2} / 6\right)\left(k_{B} T / \Delta\right)^{2}$, at low temperature, which is consistent with a numerical simulation 26]. If we extrapolate this relation to the critical temperature $T_{c}$ at which $L^{z}\left(T_{c}\right)$ vanishes, we obtain the angular momentum of the ground state, up to a numerical factor, $L^{z}(T=0) \sim-\hbar\left(\pi k_{F}^{2} / 2\right)\left(k_{B} T_{c} / \Delta\right)^{2} \sim-\hbar n / 2$, indicative of $\gamma=0$. (Here we should keep in mind that we used $\kappa_{H} \propto T$ which is, however, valid only at low temperature $T \ll T_{c}$.) Since the chiral Majorana edge modes of a 2d-TSC are moving in the opposite direction to the rotation of Cooper pairs [Fig. 11(b)], at finite temperature, they contribute to reduce the total angular momentum from its ground state value at $T=0$.

Possible experiments We conclude by discussing possible experiments to probe the cross-correlated response of TSCs. For the $2 \mathrm{~d}$ case, let us assume a $2 \mathrm{~d}-\mathrm{TSC}$ is rotating with frequency $\Omega^{z}$. As $\Omega^{z}$ increases by $\Delta \Omega^{z}$, we predict the temperature changes as $\Delta T=\Delta Q / C=$ $\left(2 \kappa_{H} T / C v^{2}\right) \Delta \Omega^{z}$, where $C$ is the heat capacity of the 2d-TSC sample. As the heat capacity of a fully gapped superconductor is small [6], this temperature change may not be so difficult to measure.

For 3d-TSCs, the Einstein-de Hass effect will reveal a relationship between magnetism and angular momentum of the system, as it has been used for the study of ferromagnetic materials. We assume a cylindrical 3d-TSC suspended by a thin string and apply thermal gradient
[Fig. [1(d)]. This induces surface energy current with angular momentum $L^{z}$, according to Eq. (11). By the conservation law of total angular momentum, it must be compensated by a mechanical angular momentum of the material, which can be directly measured in principle. Its inverse effect is the generation of thermal polarization (entropy polarization) by rotating the system. To observe the effect, the frequency $\Omega^{z}$ should be lower than the critical frequency $\Omega_{c 1}$ above which vortices are introduced in the bulk of the sample that will generate the energy current in the $z$ direction. The key point is that magnetization has to be induced properly on the surface, all pointing out or in 24.

In summary, we have found nontrivial correlated responses of TSCs to thermal gradient and mechanical rotation which are analogous to the topological ME effect. We have proposed possible experiments which probe these topological responses.

While we were finalizing the paper, a preprint 27] appeared in which the energy magnetization $M_{E}$ is discussed while its relation to the angular momentum $L$ and TSCs are not discussed. This work is supported by MEXT Grand-in-Aid No. 20740167, 19048008, 19048015, 21244053, Strategic International Cooperative Program (Joint Research Type) from Japan Science and Technology Agency, and by the Japan Society for the Promotion of Science (JSPS) through its "Funding Program for World-Leading Innovative R\&D on Science and Technology (FIRST Program)."

[1] The Quantum Hall Effect, edited by R. E. Prange and S. M. Girvin (Springer, New York, 1987).

[2] M. Z. Hasan and C. L. Kane, Rev. Mod. Phys. 82, 3045 (2010); X.-L. Qi and S.-C. Zhang, Rev. Mod. Phys. 83, 1057 (2011).

[3] D. J. Thouless et al., Phys. Rev. Lett. 49, 405 (1982).

[4] Quantum Liquids: Bose Condensation and Cooper Pairing in Condensed-Matter Systems, A. J. Leggett (Oxford University Press, USA), (2006).

[5] The Universe in a Helium Droplet, G. E. Volovik (Oxford University Press, USA), (2003).

[6] A. P. Mackenzie and Y. Maeno, Rev. Mod. Phys. 75, 657 (2003).

[7] G. Moore and N. Read, Nucl. Phys. B 360, 362 (1991).

[8] N. Read and D. Green, Phys. Rev. B 61, 10267 (2000).

[9] A. P. Schnyder, S. Ryu, A. Furusaki, and A. W. W. Ludwig, Phys. Rev. B 78, 195125 (2008).

[10] A. Yu Kitaev, AIP Conf. Proc. 1134, 22 (2009).

[11] X.-L. Qi, T. L. Hughes, and S.-C. Zhang, Phys. Rev. B 78, 195424 (2008).

[12] A. M. Essin, J. E. Moore, and D. Vanderbilt, Phys. Rev. Lett. 102, 146805 (2009).

[13] We do not distinguish TSFs from TSCs henceforth, because thermal responses are generic and common to both of them.

[14] Y. S. Hor et al., Phys. Rev. Lett. 104, 057001 (2010). 
[15] L. Fu and E. Berg, Phys. Rev. Lett. 105, 097001 (2010).

[16] S. Ryu, J. E. Moore, and A. W. W. Ludwig, arXiv:1010.0936.

[17] Z. Wang, X.-L. Qi, and S.-C. Zhang, Phys. Rev. B 84, 014527 (2011) [arXiv:1011.0586].

[18] P. Strěda, J. Phys. C: Solid State Phys. 15, 717 (1982).

[19] D. Lynden-Bell and M. Nouri-Zonoz, Rev. Mod. Phys. 70, 427 (1998).

[20] J. M. Luttinger, Phys. Rev. 135, 1505 (1964).

[21] See supplemental material.

[22] S. Murakawa et al., J. Phys. Soc. Jpn. 80, 013602 (2011).

[23] L. Smrčka and P. Strěda, J. Phys. C: Solid State Phys. 10, 2153 (1977).

[24] It may not be an easy task to realize this setup. However, when both rotation and temperature gradient are introduced simultaneously, local spins near the surface will spontaneously align to lower the total energy by gaining the coupling energy $U_{\theta}$ given in Eq. 114, as in the case of 3d-TIs, K. Nomura and N. Nagaosa, Phys. Rev. Lett. 106, 166802 (2011).

[25] G. E. Volovik and A. Vilenkin, Phys. Rev. D 62, 025014 (2000).

[26] T. Kita, J. Phys. Soc. Jpn. 67, 216 (1998).

[27] T. Qin, Q. Niu, and J. Shi, arXiv:1108.3879.

\section{THERMAL STRĚDA FORMULA FROM THE CFT PARTITION FUNCTION}

In this appendix, we will derive the thermal Strěda formula (5) of $2 \mathrm{~d}$ topological liquid by making use of the partition function of the chiral conformal field theory (CFT) on the edge. Our derivation closely follows the calculations of the thermal conductivity, the Coulomb blockade current peak, and the thermopower by Cappelli et al 113 .

While the Strěda formula can be derived solely from the Kubo formula in the bulk, as illustrated in the text in terms of the $2 \mathrm{~d}$ Majorana fermion system, the purpose of such complementary derivation is two-fold: (i) In the bulk of a topological fluid, the dispersion of gapped quasiparticle excitations is not universal and one can adiabatically deform it without changing the topological properties of the system. On the other hand, the dispersion of gapless excitations on the edge is, at low energies, quite universal with emergent Lorentz invariance.

(ii) While the derivation in terms of the Kubo formula mainly focuses, at least in the form presented in the main text, the situation where there is no interaction among quasiparticles, the derivation in term of the edge theory applies to an arbitrary (chiral) topological liquid, including strongly interacting ones such as the fractional quantum Hall liquid.

For the Strěda formula for the electrical Hall conductivity in the quantum Hall systems, and for the spin Hall conductivity in the quantum spin Hall systems, an approach similar to the calculations we present in this section can be developed. Below, we will set $\hbar=c=k_{B}=$ 1 .

\section{partition function}

Let us consider a topological fluid in the annulus geometry which is boundary by two (inner and outer) edges. The low-energy physics is governed by the two edge modes. The Hamiltonian for the edge modes may be written as

$$
H=\frac{v_{1}}{R_{1}}\left(L_{0}-\frac{c}{24}\right)+\frac{v_{2}}{R_{2}}\left(\bar{L}_{0}-\frac{c}{24}\right),
$$

where $L_{0}$ and $\bar{L}_{0}$ represents the energy-momentum tensor for the outer and inner edges, respectively; $v_{1}$ and $v_{2}$ are the fermi velocity for the outer and inner edges, respectively; $R_{1}$ and $R_{2}$ are the circumference of the outer and inner edges, respectively; By "adjusting" the velocities of propagation of excitations, we may set $v_{1} / R_{1}=$ $v_{2} / R_{2} \equiv v / R$.

We now consider the grand canonical partition function for the edges defined by

$$
Z=\operatorname{Tr} e^{-\beta\left[H-\Omega^{z} L_{z}-\mu(N+\bar{N})-V_{o}(N-\bar{N})\right]},
$$

where we have included the frequency $\Omega^{z}$, the chemical potential $\mu$, and the electro potential difference between edges $V_{o}$; they couple to the total orbital angular momentum, the total electron number, and the difference between the electron numbers in the inner and outer edges; $N$ and $\bar{N}$ represent the total electric charge of the inner and out edges, respectively.

Below, we will organize these intensive variables in terms of two complex numbers $\tau$ and $\zeta$,

$$
2 \pi i \tau=-\beta \frac{v+i \Omega^{z} /(\pi R)}{R}, \quad 2 \pi i \zeta=-\beta\left(V_{o}+i \mu\right),
$$

where we have "analytically continued" $\Omega^{z} \rightarrow i \Omega^{z}$, and $\mu \rightarrow i \mu$. The grand partition function $Z(\tau, \zeta)$ is then given by

$$
Z(\tau, \zeta)=\operatorname{tr}\left[e^{2 \pi i \tau\left(L_{0}-c / 24\right)-2 \pi i \bar{\tau}\left(\bar{L}_{0}-c / 24\right)+\zeta N+\bar{\zeta} \bar{N}}\right] .
$$

In general, for a rational topological phase, where the number of types of bulk quasiparticles is finite, the annulus partition function can be computed as

$$
Z(\tau, \zeta)=\sum_{a, \bar{b}} n_{a, \bar{b}} \chi_{a}(\tau, \zeta) \overline{\chi_{b}^{c}(\tau, \zeta)}
$$

where $\chi_{a}(\tau, \zeta)$ is a character associated with the quasiparticle of type $a, \cdots$ is complex conjugation, $\cdots^{c}$ represents particle-hole conjugation, $n_{a, \bar{b}}$ is a non-negative integer, and the summation runs over a finite set of quasiparticle types. More specifically, the character $\chi_{a}(\tau, \zeta)$ is given by

$$
\chi_{a}(\tau, \zeta):=\operatorname{tr}_{a}\left[e^{2 \pi i \tau\left(L_{0}-c / 24\right)+\zeta N}\right],
$$


where $\operatorname{tr}_{a}[\cdots]$ represents the trace within the Hilbert space associated to the particle type $a$.

On the disk geometry bounded by only one edge, the partition function does not consists of holomorphic and antiholomorphic parts, but reduces to

$$
Z(\tau, \zeta)=\chi_{a}(\tau, \zeta)
$$

where the label $a$ depends on quasiparticles present in the bulk. In particular, in the absence of quasiparticles, we have $a=0$ which represents the identity or "vacuum" particle.

\section{Strěda formula}

In order to compute the thermal Hall conductivity, $\kappa_{H}=\partial J_{E} / \partial T$, we focus on the case with single edge (i.e., disk geometry), and compute the expectation value of the energy current $J_{E}$, which can be written as

$$
J_{E}=v \varepsilon
$$

where $\varepsilon$ is the average chiral energy density on a single edge and $v$ is the velocity of the edge excitations. This can be computed from the chiral partition function (21) as

$$
\varepsilon=\frac{i v}{2 \pi R \beta} \frac{\partial}{\partial \eta} \ln \chi_{a},
$$

where we have introduced $\eta$

$$
\eta:=i \Omega^{z} /(\pi R)
$$

for notational convenience. (Henceforth we set $\zeta=\bar{\zeta}=$ $0)$. The thermal Hall conductivity is then given by

$$
\kappa_{H}=\frac{i v^{2}}{2 \pi R} \frac{\partial}{\partial T} \frac{\partial}{\partial \eta} \frac{1}{\beta} \ln \chi_{a} .
$$

On the other hand, the entropy is give by

$$
S=\frac{d}{d T} \frac{1}{\beta} \ln Z=\frac{d}{d T} \frac{1}{\beta} \ln \chi_{a} .
$$

Taking the derivative with respect to $\Omega^{z}$ on the both sides,

$$
\left.\frac{v^{2}}{2} \frac{\partial S}{\partial \Omega^{z}}\right|_{T}=\frac{v^{2}}{2} \frac{i}{\pi R} \frac{\partial}{\partial \eta} \frac{\partial}{\partial T} \frac{1}{\beta} \ln \chi_{a} .
$$

We thus obtain the Strěda formula for the thermal conductivity:

$$
\kappa_{H}=\left.\frac{\partial J_{E}}{\partial T}\right|_{\Omega^{z}}=\left.\frac{v^{2}}{2} \frac{\partial S}{\partial \Omega^{z}}\right|_{T} .
$$

We now evaluate the thermal conductivity in the large size $R \rightarrow \infty$ and in the low-temperature $\beta \rightarrow \infty$ limit.
It is important to take the $R \rightarrow \infty$ limit first, so we take the limit

$$
v \beta / R \ll 1
$$

first and then take the small $T$ limit. The limit $v \beta / R \rightarrow 0$ can be evaluated by making use of the modular transformation of the characters

$$
\chi_{a}(\tau)=\sum_{b} S_{a}^{b} \chi_{b}(-1 / \tau),
$$

where $S_{a}^{b}$ is the modular $S$-matrix. The $v \beta / R \rightarrow 0$ limit of the RHS can be evaluated as

$$
\begin{aligned}
\chi_{a}(q) & =\operatorname{tr}_{a}\left[q^{L_{0}-c / 24}\right] \\
& \sim-\frac{c}{24} \ln q+\ln \left\langle a ; 0\left|q^{L_{0}}\right| a ; 0\right\rangle \\
& =\left(-\frac{c}{24}+h_{a}\right) \ln q,
\end{aligned}
$$

where we assume the modulus of the complex parameter $q:=e^{-(2 \pi i / \tau)}$ is small, $|q| \rightarrow 0$, and $|a ; 0\rangle$ is the highest weight state, and $L_{0}|a ; 0\rangle=h_{a}|a ; 0\rangle$. Thus,

$$
\chi_{a}(\tau) \sim \sum_{b} S_{a}^{b} e^{-(2 \pi i / \tau)\left(-\frac{c}{24}+h_{b}\right)} .
$$

In the limit $\operatorname{Re}(2 \pi i / \tau)=\left(4 \pi^{2} R\right) /(v \beta) \rightarrow \infty, b=0$ (the identity particle) dominates in the summation (unless $\left.S_{a}^{0} \neq 0\right)$. Thus,

$$
\ln \chi_{a}(\tau) \sim \ln S_{a}^{0}+\frac{2 \pi i}{\tau} \frac{c}{24}
$$

Extracting the temperature dependence,

$$
\varepsilon \sim-\frac{i}{2 \pi R} \frac{c}{24} \frac{\partial}{\partial \gamma} \frac{2 \pi i}{\tau}=\frac{2 \pi T^{2}}{v} \frac{c}{24} .
$$

Finally, $\kappa_{H}$ is given by

$$
\kappa_{H}=v \frac{\partial}{\partial T} \varepsilon=v \frac{\partial}{\partial T} \frac{2 \pi T^{2}}{v} \frac{c}{24}=c \frac{\pi T}{6} .
$$

Alternatively, one can obtain the entropy first:

$$
\begin{aligned}
S & =\left(1-\tau \frac{\partial}{\partial \tau}\right) \ln \chi_{a} \\
& \sim\left(1-\tau \frac{\partial}{\partial \tau}\right) \frac{c}{24} \frac{2 \pi i}{\tau}=2 \frac{c}{24} \frac{2 \pi i}{\tau}
\end{aligned}
$$

where, again, we take the limit $v \beta / R \rightarrow 0$. The entropy can be expanded in small $\eta$ as

$$
S=\frac{c \pi^{2}}{3} \frac{1}{\beta} \frac{R}{v}(1-i \eta / v)+\mathcal{O}\left(\eta^{2}\right) .
$$

We thus conclude

$$
\kappa_{H}=-\frac{v^{2}}{2} \frac{1}{\pi R} \frac{\partial S}{\partial i \eta}=\frac{c \pi}{6} T
$$

in agreement with Eq. (35). 


\section{THERMAL STRĚDA FORMULA FROM THE 2D MAJONARA HAMILTONIAN}

\section{Strěda formula}

The energy-current operator is divided into the following two terms: $(a, b=x, y)$

$$
j_{E}^{a}=j_{E(0)}^{a}+j_{E(1)}^{a},
$$

where

$$
\begin{aligned}
j_{E(0)}^{a}= & \psi^{T} \frac{1}{4}\left\{v^{a}, \mathcal{H}\right\} \psi \\
j_{E(1)}^{a}= & -\psi^{T} \frac{i \hbar}{8}\left[v^{a}, v^{b}\right] \psi \partial_{b} \phi \\
& +\psi^{T} \frac{1}{8}\left[\mathcal{H}\left(v^{a} x^{b}+3 x^{b} v^{a}\right)+\text { H.c. }\right] \psi \partial_{b} \phi
\end{aligned}
$$

and $v^{a}=(i / \hbar)\left[\mathcal{H}, x^{a}\right] . j_{E(0)}^{a}$ is non-vanishing even in the absence of gravitational potential, while $j_{E(0)}^{a}$ is proportional to $\nabla \phi$. To evaluate $\kappa_{H}=-\left\langle j_{E}^{x}\right\rangle /\left(T \partial_{y} \phi\right)$, we apply the Kubo linear response formula to $j_{E,(0)}^{a}$ :

$$
\begin{aligned}
-\frac{\left\langle j_{E(0)}^{a}\right\rangle}{\partial_{b} \phi}=- & \frac{i}{2 L^{2}} \sum_{n m} \frac{f\left(E_{n}\right)-f\left(E_{m}\right)}{E_{n}-E_{m}}\left(\frac{E_{n}+E_{m}}{2}\right)^{2} \\
& \times \frac{\left\langle n\left|v^{a}\right| m\right\rangle\left\langle m\left|v^{b}\right| n\right\rangle}{E_{n}-E_{m}+i \eta}
\end{aligned}
$$

Here $\langle\boldsymbol{x} \mid n\rangle=u_{n}(\boldsymbol{x})$ is the exact eigenstate of the Majorana Hamiltonian, $\mathcal{H} u_{n}(\boldsymbol{x})=\varepsilon_{n} u_{n}(\boldsymbol{x})$. On the other hand, $j_{E(1)}^{a}$ needs to be averaged in thermal equilibrium:

$$
\begin{aligned}
-\left\langle j_{E(1)}^{a}\right\rangle_{0}= & \frac{1}{2 L^{2}} \sum_{n} f\left(E_{n}\right) E_{n}\left\langle n\left|\left(x^{a} v^{b}-x^{b} v^{a}\right)\right| n\right\rangle \partial_{b} \phi \\
& +\frac{1}{2} \frac{1}{L^{2}} \sum_{n} f\left(E_{n}\right) \frac{i \hbar}{4}\left\langle n\left|\left[v^{a}, v^{b}\right]\right| n\right\rangle \partial_{b} \phi .
\end{aligned}
$$

One obtains the thermal Hall conductivity

$$
\kappa_{H}=\frac{1}{T} \int_{-\infty}^{\infty} d \varepsilon\left(\frac{-\partial f(\varepsilon)}{\partial \varepsilon}\right) \frac{\varepsilon^{2}}{2} \int_{-\infty}^{\varepsilon} d \zeta A(\zeta),
$$

where [4, 5,23

$$
\begin{aligned}
& A(\zeta)=\frac{1}{2} \frac{d B}{d \zeta}+\frac{1}{2 L^{2}} \operatorname{Tr}\left[\left(x v^{y}-y v^{x}\right) \frac{d \delta(\zeta-\mathcal{H})}{d \zeta}\right] \\
& B(\zeta)=\frac{i}{L^{2}} \operatorname{Tr}\left\{\left[v^{x} G_{+}(\zeta) v^{y}-v^{y} G_{-}(\zeta) v^{x}\right] \delta(\zeta-\mathcal{H})\right\}
\end{aligned}
$$

and $G_{ \pm}(\zeta)=(\zeta-\mathcal{H} \pm i \eta)^{-1}$. It is convenient to write the thermal conductivity as a sum of the following two terms:

$$
\begin{aligned}
\kappa_{H}^{I} & =\frac{1}{2 T} \int d \varepsilon \frac{\partial f(\varepsilon)}{\partial \varepsilon} \frac{\varepsilon^{2}}{2} B(\varepsilon), \\
\kappa_{H}^{I I} & =\frac{\partial}{\partial T} \int d \varepsilon f(\varepsilon) \frac{1}{4} \operatorname{Tr}\left[\varepsilon\left(x v^{y}-y v^{x}\right) \delta(\varepsilon-\mathcal{H})\right] \\
& =v \frac{\partial M_{E}^{z}}{\partial T}
\end{aligned}
$$

where

$$
M_{E}^{z}=\frac{1}{4 v} \sum_{n} f\left(\varepsilon_{n}\right) \varepsilon_{n}\left\langle n\left|\left(x v^{y}-y v^{x}\right)\right| n\right\rangle .
$$

At low temperature, the Fermi distribution function can be expanded as follows:

$$
f\left(\varepsilon_{n}-\zeta\right)=\theta\left(\zeta-\varepsilon_{n}\right)-\frac{\pi^{2}}{6} T^{2} \frac{d}{d \zeta} \delta\left(\zeta-\varepsilon_{n}\right)
$$

and thus

$$
\kappa_{H}^{I} \simeq \frac{\pi^{2} k_{B}^{2} T^{2}}{6} B(\zeta=0) .
$$

When the mass gap is large enough, the density of states of Majorana fermions and Eq. (45) vanish at $\varepsilon=0$, and hence $\kappa_{H}=\kappa_{H}^{I I}$ while $\kappa_{x x}=0$.

\section{Generalized Wiedemann-Franz law}

Here we derive Eq. (9). Noting the identity

$$
\begin{aligned}
& \int_{-\infty}^{\epsilon} d \zeta A(\zeta) \\
= & \int_{-\infty}^{\epsilon} d \zeta \frac{i}{L^{2}} \operatorname{Tr}\left[v^{x} \frac{-1}{(\zeta-\mathcal{H}+i \eta)^{2}} v^{y} \delta(\zeta-\mathcal{H})-\text { H.c. }\right] \\
= & \frac{-i}{L^{2}} \sum_{n m} \theta\left(\zeta-\epsilon_{n}\right)\left[\frac{\left\langle n\left|v^{x}\right| m\right\rangle\left\langle m\left|v^{y}\right| n\right\rangle}{\left(\epsilon_{n}-\epsilon_{m}+i \eta\right)^{2}}-\text { C.c }\right]
\end{aligned}
$$

in Eq. (44) and using Eq. (49), one obtains Eq. (9).

[1] Andrea Cappelli, Marina Huerta, and Guillermo R. Zemba, Nucl. Phys. B 636, 568 (2002).

[2] Andrea Cappelli, Giovanni Viola and Guillermo R. Zemba, Annals of Physics, 325, 465 (2010).

[3] Andrea Cappelli, and Giovanni Viola, J. Phys. A 44, 075401 (2011).

[4] O. Vafek, A. Melikyan, and Z. Tesanovic, Phys. Rev. B 64, 224508 (2001).

[5] R. Matsumoto and S. Murakami, arXiv:1106.1987. 